\title{
Serum Albumin Concentrations in Stable Chronic Obstructive Pulmonary Disease: A Systematic Review and Meta-Analysis
}

\author{
Elisabetta Zinellu ${ }^{1}$, Alessandro G. Fois ${ }^{1,2}$, Elisabetta Sotgiu ${ }^{3}$, Sabrina Mellino ${ }^{3}$, Arduino A. Mangoni ${ }^{4}$ (i),

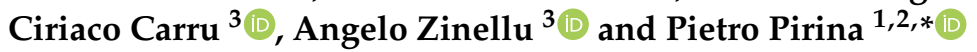 \\ 1 Unit of Respiratory Diseases, University Hospital Sassari (AOU), 07100 Sassari, Italy; \\ elisabetta.zinellu@aousassari.it (E.Z.); agfois@uniss.it (A.G.F.) \\ 2 Department of Medical, Surgical and Experimental Sciences, University of Sassari, 07100 Sassari, Italy \\ 3 Department of Biomedical Sciences, University of Sassari, 07100 Sassari, Italy; esotgiu@uniss.it (E.S.); \\ sabrinamellino3@gmail.com (S.M.); carru@uniss.it (C.C.); azinellu@uniss.it (A.Z.) \\ 4 Department of Clinical Pharmacology, College of Medicine and Public Health, Flinders University and \\ Flinders Medical Centre, Adelaide 5001, Australia; arduino.mangoni@flinders.edu.au \\ * Correspondence: pirina@uniss.it
}

check for updates

Citation: Zinellu, E.; Fois, A.G.; Sotgiu, E.; Mellino, S.; Mangoni, A.A.; Carru, C.; Zinellu, A.; Pirina, P. Serum Albumin Concentrations in Stable Chronic Obstructive Pulmonary Disease: A Systematic Review and Meta-Analysis. J. Clin. Med. 2021, 10, 269. https://doi.org/10.3390/ jcm10020269

Received: 21 October 2020 Accepted: 8 January 2021 Published: 13 January 2021

Publisher's Note: MDPI stays neutral with regard to jurisdictional clai$\mathrm{ms}$ in published maps and institutional affiliations.

Copyright: (C) 2021 by the authors. Licensee MDPI, Basel, Switzerland. This article is an open access article distributed under the terms and conditions of the Creative Commons Attribution (CC BY) license (https:// creativecommons.org/licenses/by/ $4.0 /)$.

\begin{abstract}
Background: Chronic obstructive pulmonary disease (COPD) is a progressive condition characterized by chronic airway inflammation and lung parenchyma damage. Systemic inflammation and oxidative stress also play a role in the pathogenesis of COPD. Serum albumin is a negative acute-phase protein with antioxidant effects and an important marker of malnutrition. The aim of this meta-analysis was to investigate differences in serum albumin concentrations between patients with stable COPD and non-COPD subjects. Methods: A systematic search was conducted, using the terms "albumin" and "chronic obstructive pulmonary disease" or "COPD", in the electronic databases PubMed and Web of Science, from inception to May 2020. Results: Twenty-six studies were identified on a total of 2554 COPD patients and 2055 non-COPD controls. Pooled results showed that serum albumin concentrations were significantly lower in COPD patients (standard mean difference, SMD $=-0.50,95 \% \mathrm{CI}-0.67$ to $-0.32 ; p<0.001)$. No significant differences were observed in SMD of serum albumin concentrations between COPD patients with forced expiratory volume in the 1st second (FEV1) $<50 \%$ and those with FEV1 > 50\%. Conclusions: Our systematic review and meta-analysis showed that serum albumin concentrations are significantly lower in patients with stable COPD compared to non-COPD controls. This supports the presence of a deficit in systemic anti-inflammatory and antioxidant defense mechanisms in COPD.
\end{abstract}

Keywords: COPD; albumin; inflammation; oxidative stress; malnutrition

\section{Introduction}

Chronic obstructive pulmonary disease (COPD) is a common condition characterized by persistent respiratory symptoms and airflow limitation that is not fully reversible. The airflow limitation is caused by airway disease and/or lung parenchyma destruction [1]. COPD represents a major public health concern, with a global prevalence of $11.7 \%$ in 2010 [2], and it is now the third leading cause of death worldwide [3]. It is a complex disease resulting from the interaction between genetic predisposition and environmental factors. In particular, COPD is characterized by chronic inflammation due to exposure to tobacco smoke, the main risk factor for COPD, as well as to occupational exposures, outdoor and indoor air pollution, and childhood exposure to the above-mentioned risk factors [4]. Other risk factors, including lifestyle, diet, and physical activity, may also play a role [4]. This proposition is supported by the recent observation that many people develop the disease without smoking exposure [5]. Inhalation of cigarette smoke or other noxious particles causes lung inflammation that, in patients with COPD, is amplified and even further increased during acute exacerbations [6]. The mechanism responsible 
for the excess inflammatory response is not yet understood, although oxidative stress may play an important role [7]. Oxidants contained in cigarette smoke and in other inhaled particulates, as well as those produced by activated inflammatory cells, might overcome the antioxidant defense systems, leading to oxidative stress. Human serum albumin (HSA) is a multifunctional plasma protein that accounts for over $50 \%$ of the total plasma protein content. Physiologically, HSA exists predominantly in a reduced state containing a free cysteine residue (Cys34) [8]. This residue constitutes the largest pool of thiols in the peripheral blood and represents the main pathway through which albumin scavenges reactive oxygen and nitrogen species (ROS and RNS) [9,10]. In addition to its antioxidant properties, albumin is also a negative acute phase reactant, and its concentrations decrease during the acute phase response [11]. Moreover, albumin is an established clinical marker of malnutrition [12]. Malnutrition is common among COPD patients and negatively impacts on quality of life, risk of exacerbations, length of hospital stay, and overall healthcare costs [13]. Similarly, hypoalbuminemia has been associated with a prolonged length of hospital stay during acute exacerbations, acute respiratory failure, and increased mortality in patients with COPD [14-16]. Several studies have investigated serum albumin concentrations in patients with stable COPD and non-COPD subjects, with some conflicting results. We sought to address this issue by conducting a systematic review and meta-analysis in order to investigate the presence and the effect size of differences in serum albumin concentrations between the two groups.

\section{Experimental Section}

\subsection{Search Strategy, Eligibility Criteria, and Study Selection}

A systematic search, using the terms "albumin" and "chronic obstructive pulmonary disease" or "COPD", was conducted in the electronic databases PubMed and Web of Science from inception to May 2020. The references of the retrieved articles and reviews were also cross-checked to identify additional studies. Eligibility criteria were as follows: (i) assessment of serum or plasma albumin; (ii) comparison of subjects with or without COPD (case-control design); (iii) >10 subjects investigated; and (iv) articles in English. Two investigators (ES and SM) independently screened individual abstracts. If relevant, they independently reviewed the full articles; any disagreement was resolved by a third investigator (AZ). The Newcastle-Ottawa scale was used to assess the quality of each study by evaluating the following components: cohort selection, cohort comparability on the basis of the design or analysis, how the exposure was determined, and how the outcomes of interest were evaluated [17]. Studies achieving a score of six or more were considered to be of high quality.

\subsection{Statistical Analysis}

Standardized mean differences (SMD) were used to build forest plots of continuous data and to evaluate differences in serum albumin concentrations between non-COPD controls and COPD patients. A $p$-value $<0.05$ was considered statistically significant, and $95 \%$ confidence intervals (CIs) were reported. In two studies [18,19], the median and interquartile range (IQR) values were extracted from a graph by the Graph Data Extractor program, and the mean and standard deviation values were calculated from the median and IQR, as previously described [20]. Q statistic was used to test the heterogeneity of SMD across studies (significance level at $p<0.10$ ). Inconsistency across studies was evaluated through the $\mathrm{I}^{2}$ statistic $\left(\mathrm{I}^{2}<25 \%\right.$, no heterogeneity; $\mathrm{I}^{2}$ between $25 \%$ and $50 \%$, moderate heterogeneity; $\mathrm{I}^{2}$ between $50 \%$ and $75 \%$, large heterogeneity; and $\mathrm{I}^{2}>75 \%$, extreme heterogeneity) $[21,22]$. A random-effects model was used to calculate the pooled SMD and corresponding 95\% confidence intervals, due to high heterogeneity. The influence of each individual study on the overall risk estimate was investigated through sensitivity analysis, by sequentially excluding one study in each turn [23]. The associations between study size and magnitude of effect were analyzed by means of Begg's adjusted rank correlation test and Egger's regression asymmetry test at the $p<0.05$ level of significance 
to assess the presence of potential publication bias [24,25]. We also performed the Duval and Tweedie "trim and fill" procedure [26] to further test the possible effect of publication bias. This method recalculates a pooled SMD by incorporating the hypothetical missing studies as though they actually existed, to augment the observed data so that the funnel plot is more symmetric. Statistical analyses were performed using Stata 14 (STATA Corp., College Station, TX, USA). The study was fully compliant with the PRISMA statement for reporting of systematic reviews and meta-analyses [27].

\section{Results}

A flow chart depicting the screening process is described in Figure 1. We initially identified 1329 studies. A total of 1283 studies were excluded because they were either duplicates or irrelevant. After a full-text revision of 46 articles, 20 studies were excluded because they did not meet the inclusion criteria. Thus, twenty-six studies were included in the meta-analysis $[18,19,28-51]$. The characteristics of the retrieved studies, published between 1994 and 2020, are presented in Table 1. A total of 2554 COPD patients (74\% males) and 2055 non-COPD controls (63\% males) were evaluated. Overall, the mean age of participants across all studies was 61.7 years in COPD patients and 64.6 years in controls. Almost all (24 out of 26) were prospective studies. In sixteen studies, the diagnosis of COPD was made according to the Global Obstructive Lung Disease (GOLD) guidelines, whilst the American Thoracic Society (ATS) guidelines were used in five, the European Respiratory Society (ERS) guidelines were used in four, and the Spanish COPD Guidelines (GesEPOC) were used in one. Significant differences in serum albumin concentrations between COPD patients and control subjects were reported in $50 \%$ of the selected studies. The forest plot for serum albumin concentrations in COPD patients and controls is shown in Figure 2. Due to the extreme heterogeneity between studies $\left(\mathrm{I}^{2}=85.7 \%, p<0.001\right)$, random-effects models were used to perform the analysis. Pooled results showed that serum albumin concentrations were significantly lower in COPD patients (SMD $=-0.50,95 \% \mathrm{CI}-0.67$ to $-0.32 ; p<0.001)$. Sensitivity analysis, reported in Figure 3, showed that the effect size was not modified when any single study was in turn removed (effect size ranged between -0.44 and -0.52$)$. The Begg's $(p=0.04)$ and Egger's tests $(p=0.01)$ showed a significant publication bias; however, the trim-and-fill analysis found that no study was missing or should be added (Figure 4).

In 23 studies, the control group comprised healthy subjects. In the remaining three, it consisted of subjects hospitalized for other medical conditions or some type of surgery [28], patients with diseases other than COPD [43], or lung cancer patients [48]. The SMD was not affected $(-0.53,95 \% \mathrm{CI}-0.72$ to $-0.33, p<0.001 ; \mathrm{I} 2=86.1 \%, p<0.001)$ after removing these studies from the meta-analysis.

We also investigated the effects of disease severity (forced expiratory volume in the 1st second FEV1 $<50 \%$ or FEV1 $>50 \%$ ) and the guidelines used for diagnosis. No significant differences $(p=0.73)$ were observed in SMD of serum albumin concentrations between subjects with FEV1 < 50\% (SMD $=-0.46,95 \% \mathrm{CI}-0.71$ to $-0.21, p<0.001 ; \mathrm{I}^{2}=86.6 \%$, $p<0.001)$ and those with FEV1 $>50 \%(\mathrm{SMD}=-0.57,95 \% \mathrm{CI}-0.88$ to $-0.26 \mathrm{~g} / \mathrm{L}, p<0.001$; $\mathrm{I}^{2}=87.2 \%, p<0.001$ ) (Figure 5A). Analysis based on specific guidelines (Figure 5B) also indicated similar pooled SMD values between studies recruiting by ERS (SMD $=-0.57$, $95 \% \mathrm{CI}-1.05$ to $-0.10, p=0.017$ ) and GOLD guidelines (SMD $=-0.58,95 \% \mathrm{CI}-0.82$ to $-0.33, p<0.001)$ with a slight improvement in heterogeneity that may be classified as large in the ERS group $\left(\mathrm{I}^{2}=67.9 \%, p=0.025\right)$ and extreme in the GOLD group $\left(\mathrm{I}^{2}=90 \%\right.$, $p<0.001)$. In the sub-group of studies using ATS guidelines for COPD diagnosis, we found that serum albumin concentrations were not significantly different between COPD patients and control subjects (SMD $=-0.21,95 \% \mathrm{CI}-0.55$ to $0.13, p<0.124 ; \mathrm{I} 2=37.6 \%, p<0.171$ ). Then, we investigated age, sex, body mass index (BMI), FEV1, FEV1/forced vital capacity (FVC), C-reactive protein (CRP), and continent where the study was conducted (Europe, Africa, Asia, and America) and publication year as possible contributors to between-study variance. However, none of these variables was found to be significantly related to pooled 
SMD by meta-regression analysis (age $(t=-0.81, p=0.429)$, sex $(t=0.08, p=0.940)$, BMI $(t=1.37, p=0.187), \operatorname{FEV} 1(t=0.04, p=0.965), \mathrm{FEV} 1 / \mathrm{FVC}(t=0.55, p=0.591), \mathrm{CRP}(t=-0.60$, $p=0.560)$, continent $(t=0.87, p=0.393)$ and publication year $(t=-1.18, p=0.250))$.

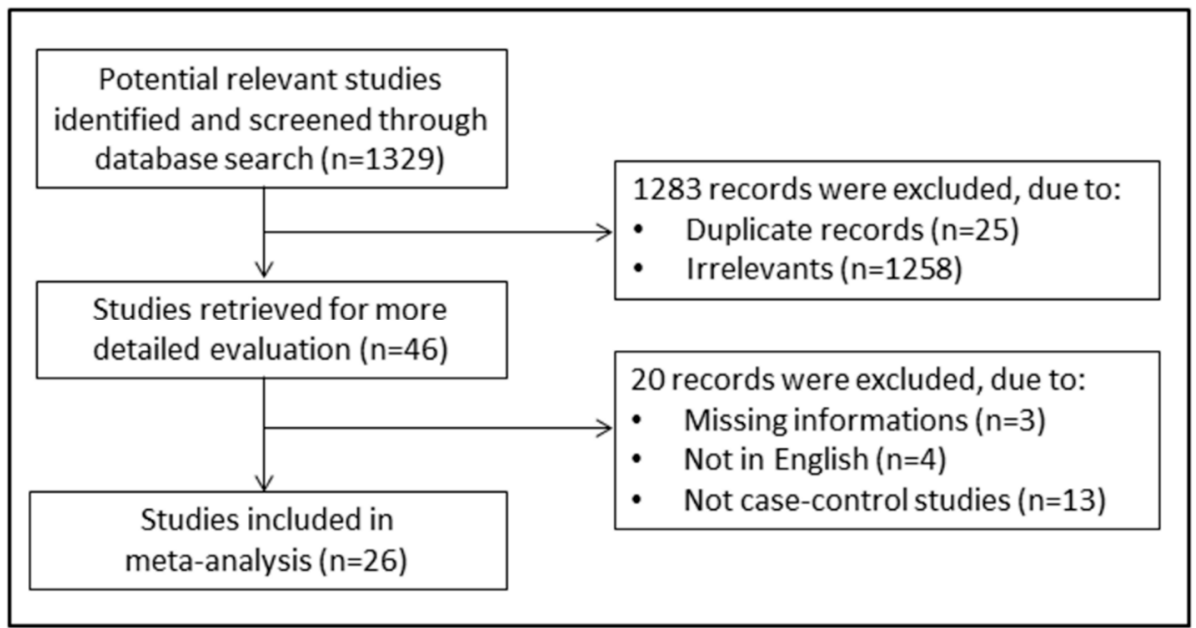

Figure 1. Flow chart of study selection.

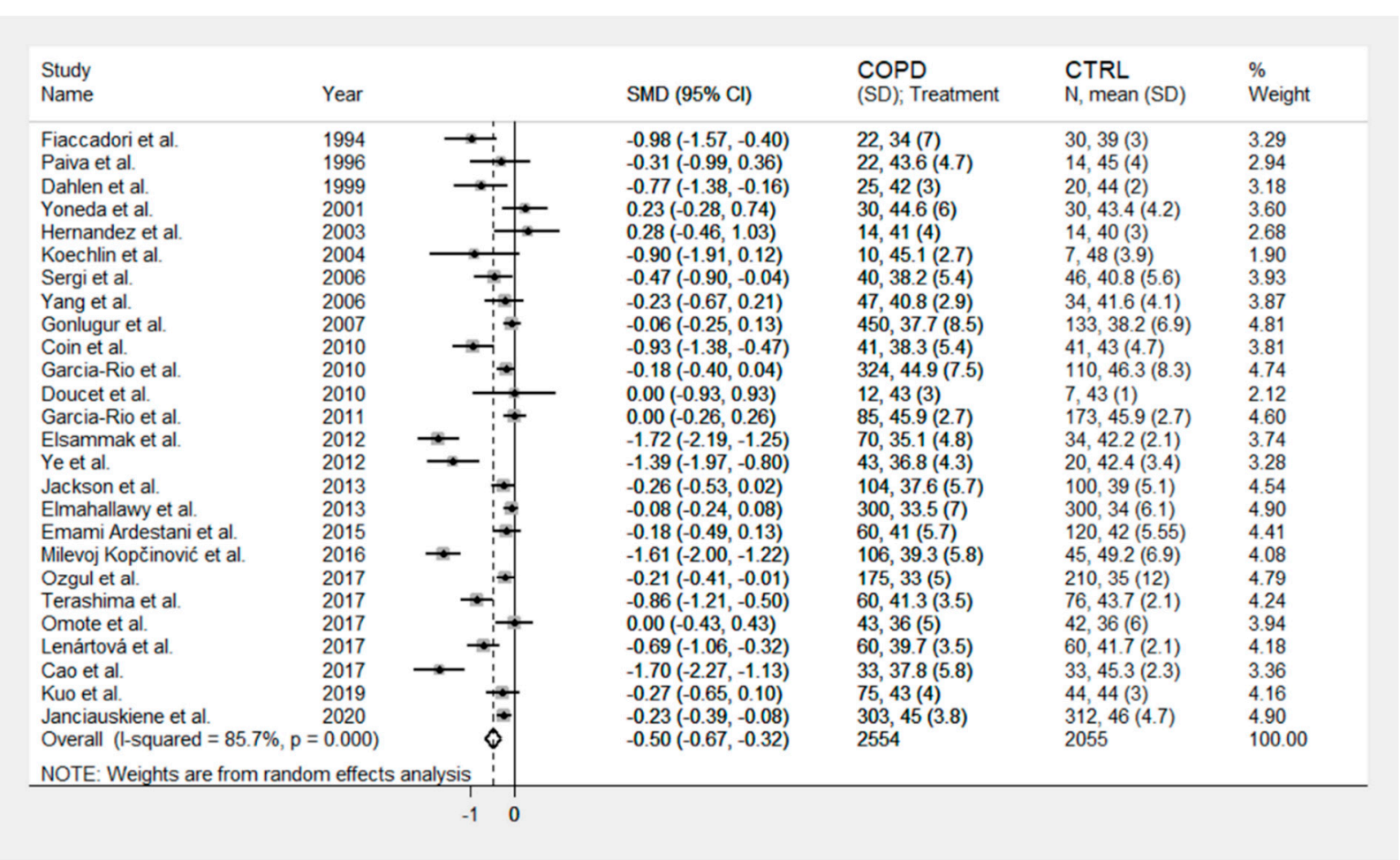

Figure 2. Forest plot of studies examining serum albumin in chronic obstructive pulmonary disease (COPD). 


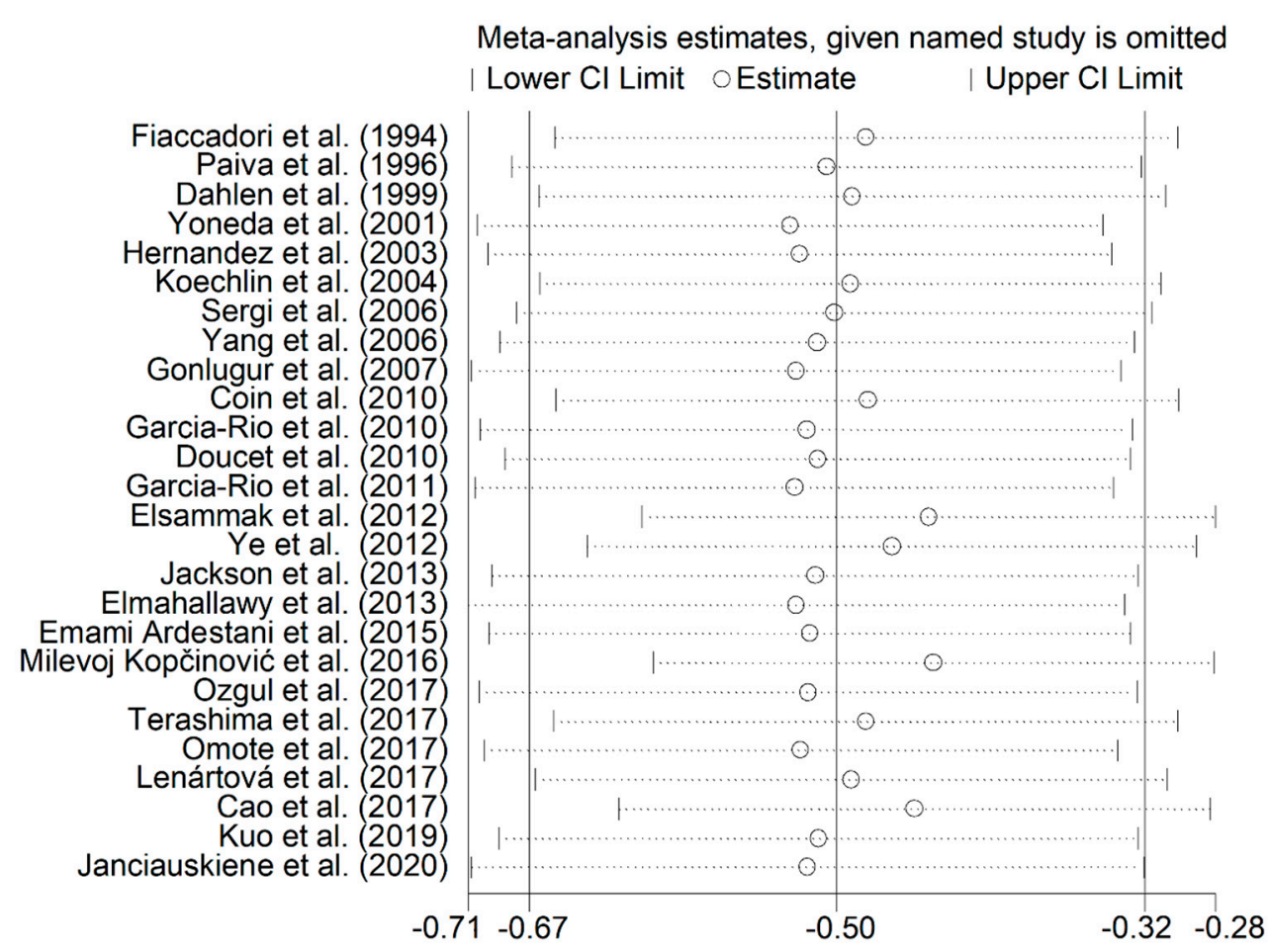

Figure 3. Sensitivity analysis of the association between albumin and COPD. The influence of individual studies on the overall standardized mean difference (SMD) is shown. The middle vertical axis indicates the overall SMD, and the two vertical axes indicate the $95 \%$ confidence intervals (CI). Hollow circles represent the pooled SMD when the remaining study is omitted from the meta-analysis. Two ends of each broken line represent $95 \%$ CI.

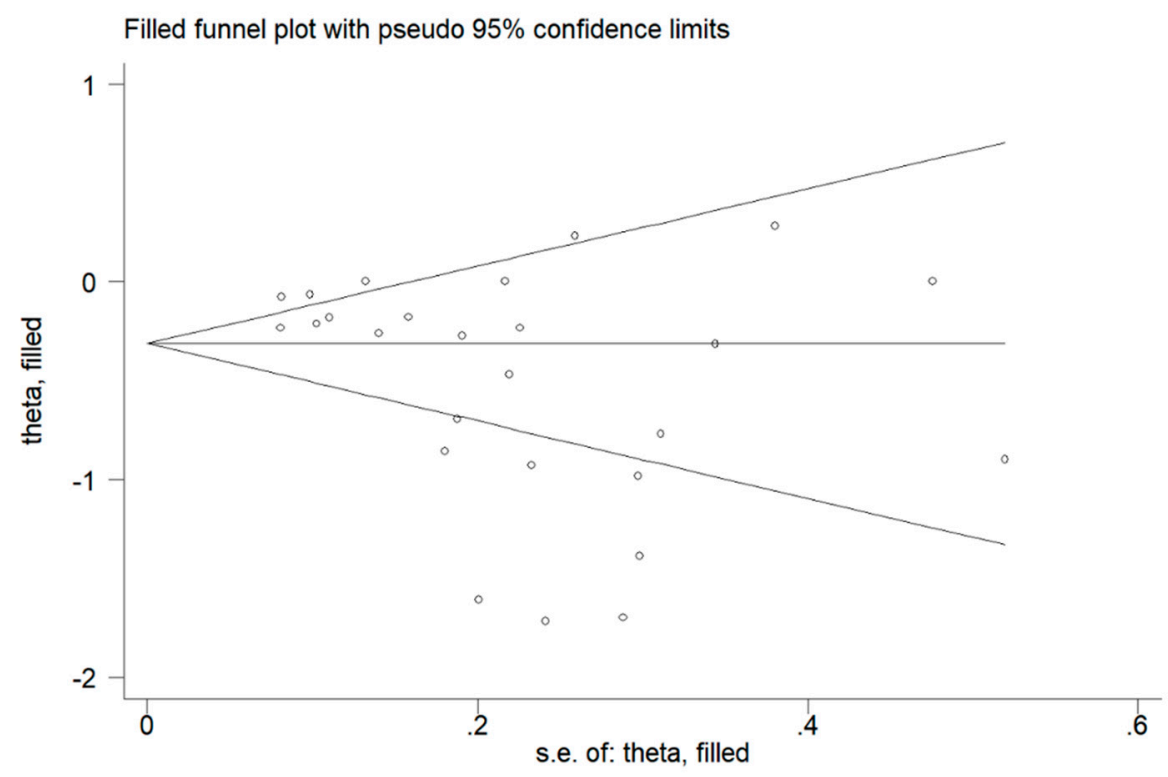

Figure 4. Funnel plot of studies investigating non-COPD controls and patients with COPD after trimming and filling. Dummy studies and genuine studies are represented by enclosed circles and free circles, respectively. 


\begin{tabular}{|c|c|c|c|c|}
\hline $\begin{array}{l}\text { Study } \\
\text { Name }\end{array}$ & Year & $\mathbf{A}$ & $\operatorname{SMD}(95 \% \mathrm{Cl})$ & \%eight \\
\hline $\begin{array}{l}\text { FEV1 not reported } \\
\text { Fiaccadori et al. } \\
\text { Paiva et al. } \\
\text { Garcia-Rio et al. } \\
\text { Subtotal (1-Squared }=78.5 \%, D=0.009 \text { ) }\end{array}$ & $\begin{array}{l}1994 \\
1996 \\
2011\end{array}$ & & $\begin{array}{l}-0.98(-1.57,-0.40) \\
-0.31(-0.99,0.36) \\
0.00(-0.26,0.26) \\
-0.40(-1.02,0.22)\end{array}$ & $\begin{array}{l}3.29 \\
2.94 \\
4.60 \\
10.82\end{array}$ \\
\hline 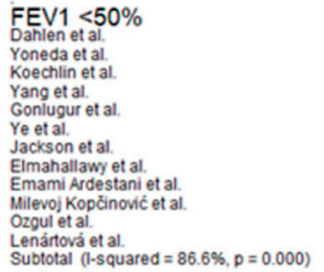 & $\begin{array}{l}1999 \\
2001 \\
2004 \\
2006 \\
2007 \\
2012 \\
2013 \\
2013 \\
2015 \\
2016 \\
2017 \\
2017\end{array}$ & & $\begin{array}{l}-0.77(-1.38,-0.16) \\
0.23(-0.28,0.74) \\
-0.90(-1.91,0.12) \\
-0.23(-0.67,0.21) \\
-0.06(-0.25,0.13) \\
-1.39(-1.97,-0.80) \\
-0.26(-0.53,0.02) \\
-0.08(-0.24,0.03) \\
-0.18(-0.49,0.13) \\
-1.61(-2.00,-1.22) \\
-0.21(-0.41,-0.01) \\
-0.69(-1.06,-0.32) \\
-0.46(-0.71,-0.21)\end{array}$ & $\begin{array}{l}3.18 \\
3.60 \\
1.90 \\
3.87 \\
4.81 \\
3.28 \\
4.54 \\
4.90 \\
4.41 \\
4.08 \\
4.79 \\
4.18 \\
47.54\end{array}$ \\
\hline $\begin{array}{l}\text { FEV1 }>50 \% \\
\text { Hernandez et al. } \\
\text { Sergi et al. } \\
\text { Coin et al. } \\
\text { Garcia-Rio et al. } \\
\text { Doucet et al. } \\
\text { Elsammak et al. } \\
\text { Terashima et al. } \\
\text { Omote et al. } \\
\text { Cao et al. } \\
\text { Kuo et al. } \\
\text { Janciausiene ot al. } \\
\text { Subtotal (l-squared }=87.2 \%, p=0.000)\end{array}$ & $\begin{array}{l}2003 \\
2006 \\
2010 \\
2010 \\
2010 \\
2012 \\
2017 \\
2017 \\
2017 \\
2019 \\
2020\end{array}$ & & $\begin{array}{l}0.28(-0.46,1.03) \\
-0.47(-0.90,-0.04) \\
-0.93(-1.38,-0.47) \\
-0.18(-0.40,0.04) \\
0.00(-0.93,0.93) \\
-1.72(-2.19,-1.25) \\
-0.86(-1.21,-0.50) \\
0.00(-0.43,0.43) \\
-1.70(-227,-1.13) \\
-0.27(-0.65,0.10) \\
-0.23(-0.39,-0.08) \\
-0.57(-0.88,-0.26)\end{array}$ & $\begin{array}{l}2.68 \\
3.93 \\
3.81 \\
4.74 \\
2.12 \\
3.74 \\
4.24 \\
3.94 \\
3.36 \\
4.16 \\
4.90 \\
41.63\end{array}$ \\
\hline $\begin{array}{l}\text { Overall (1-squared }=85.7 \%, p=0.000 \text { ) } \\
\text { NOTE: Weights are trom random effects a }\end{array}$ & analysis & 8 & $-0.50(-0.67,-0.32)$ & 100.00 \\
\hline & & 0 & & \\
\hline $\begin{array}{l}\text { Study } \\
\text { Name }\end{array}$ & Year & B & SMD (95\% Cl) & $\stackrel{\%}{\text { Weight }}$ \\
\hline $\begin{array}{l}\text { ERS } \\
\text { Fiaccadori et al. } \\
\text { Hernandez et ai. } \\
\text { Sergi et al. } \\
\text { Coin et al. } \\
\text { Subtotal (1-squared }=67.9 \%, D=0.025 \text { ) }\end{array}$ & $\begin{array}{l}1994 \\
2003 \\
2006 \\
2010\end{array}$ & & $\begin{array}{l}-0.98(-1.57,-0.40) \\
0.28(-0.46,1.03) \\
-0.47(-0.90,-0.04) \\
-0.93(-1.38,-0.47) \\
-0.57(-1.05,-0.10)\end{array}$ & $\begin{array}{l}3.29 \\
2.68 \\
3.93 \\
3.81 \\
13.71\end{array}$ \\
\hline 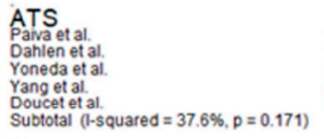 & $\begin{array}{l}1996 \\
1999 \\
2001 \\
2006 \\
2010\end{array}$ & 我 & $\begin{array}{l}-0.31(-0.99,0.36) \\
-0.77(-1.38,-0.16) \\
0.23(-0.28,0.74) \\
-0.23(-0.67,0.21) \\
0.00(-0.93,0.93) \\
-0.21(-0.55,0.13)\end{array}$ & $\begin{array}{l}2.94 \\
3.18 \\
3.60 \\
3.87 \\
2.12 \\
15.70\end{array}$ \\
\hline 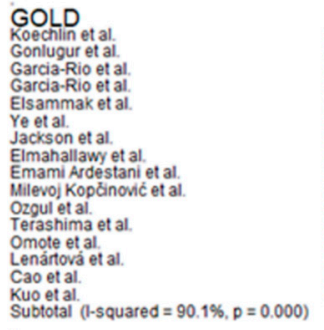 & $\begin{array}{l}2004 \\
2007 \\
2010 \\
2011 \\
2012 \\
2012 \\
2013 \\
2013 \\
2015 \\
2016 \\
2017 \\
2017 \\
2017 \\
2017 \\
2017 \\
2019\end{array}$ & : & $\begin{array}{l}-0.90(-1.91,0.12) \\
-0.06(-0.25,0.13) \\
-0.18(-0.40,0.04) \\
0.00(-0.26,0.26) \\
-1.72(-2.19,-1.25) \\
-1.39(-1.97,-0.80) \\
-0.26(-0.53,0.02) \\
-0.08(-0.24,0.08) \\
-0.18(-0.49,0.13) \\
-1.61(-2.00,-1.22) \\
-0.21(-0.41,-0.01) \\
-0.86(-1.21,-0.50) \\
0.00(-0.43,0.43) \\
-0.69(-1.06,-0.32) \\
-1.70(-227,-1.13) \\
-0.27(-0.65,0.10) \\
-0.58(-0.82,-0.33)\end{array}$ & $\begin{array}{l}1.90 \\
4.81 \\
4.74 \\
4.60 \\
3.74 \\
3.28 \\
4.54 \\
4.90 \\
4.41 \\
4.08 \\
4.79 \\
4.24 \\
3.94 \\
4.18 \\
3.36 \\
4.16 \\
65.68\end{array}$ \\
\hline $\begin{array}{l}\text { GesEPOC } \\
\text { Janclauskiene et al. } \\
\text { Subtotal }(1-s \text { quared }=\%, p=\text {.) }\end{array}$ & 2020 & 姑 & $\begin{array}{l}-0.23(-0.39,-0.08) \\
-0.23(-0.39,-0.08)\end{array}$ & $\begin{array}{l}4.90 \\
4.90\end{array}$ \\
\hline \multicolumn{2}{|l|}{$\begin{array}{l}\text { Overall (1-square } d=85.7 \%, p=0.000 \text { ) } \\
\text { NOTE: Weights are from random effects ana }\end{array}$} & \$ & $-0.50(-0.67,-0.32)$ & 100.00 \\
\hline & & 0 & & \\
\hline
\end{tabular}

Figure 5. Forest plot of studies examining albumin and COPD according to disease severity evaluated by $\mathrm{FEV}_{1}(\mathbf{A})$ and guidelines used for the diagnosis (B). 
Table 1. Summary of the studies on COPD vs. controls included in the meta-analysis.

\begin{tabular}{|c|c|c|c|c|c|c|c|c|c|c|c|}
\hline \multirow[b]{2}{*}{$\begin{array}{l}\text { First Author, } \\
\text { Year Country }\end{array}$} & \multirow[b]{2}{*}{$\begin{array}{c}\text { Study } \\
\text { Design }\end{array}$} & \multirow[b]{2}{*}{ Diagnosis } & \multirow[b]{2}{*}{$\begin{array}{l}\text { NOS } \\
\text { (Stars) }\end{array}$} & \multicolumn{4}{|c|}{ Control Group } & \multicolumn{4}{|c|}{ COPD Group } \\
\hline & & & & $n$ & $\begin{array}{l}\text { Age } \\
\text { (Years) }\end{array}$ & $\begin{array}{l}\text { Gender } \\
(\mathrm{M} / \mathrm{F})\end{array}$ & $\begin{array}{c}\text { Albumin } \\
\text { Mean } \pm \text { SD }\end{array}$ & $n$ & $\begin{array}{l}\text { Age } \\
\text { (Years) }\end{array}$ & $\begin{array}{l}\text { Gender } \\
(\mathrm{M} / \mathrm{F})\end{array}$ & $\begin{array}{c}\text { Albumin } \\
\text { Mean } \pm \text { SD }\end{array}$ \\
\hline $\begin{array}{l}\text { Fiaccadori, } \\
1994 \text { Italy, [28] }\end{array}$ & $\mathrm{P}$ & ERS & 7 & 30 & 60 & $25 / 5$ & $39 \pm 3$ & 22 & 63 & $19 / 3$ & $34 \pm 7$ \\
\hline $\begin{array}{c}\text { Paiva, } \\
1996 \text { Brazil, [29] }\end{array}$ & $\mathrm{P}$ & ATS & 7 & 14 & 52.8 & $14 / 0$ & $45 \pm 4$ & 22 & 59.4 & $22 / 0$ & $43.6 \pm 4.7$ \\
\hline $\begin{array}{c}\text { Dahlen, } \\
1999 \text { Sweden, [30] }\end{array}$ & $\mathrm{P}$ & ATS & 6 & 20 & 66 & $4 / 16$ & $44 \pm 2$ & 25 & 42 & $15 / 10$ & $42 \pm 3$ \\
\hline $\begin{array}{c}\text { Yoneda, } \\
\text { 2001 Japan, [31] }\end{array}$ & $\mathrm{P}$ & ATS & 7 & 30 & 64 & $29 / 1$ & $44.6 \pm 6.0$ & 30 & 64 & $29 / 1$ & $43.4 \pm 4.2$ \\
\hline $\begin{array}{c}\text { Hernandez, } \\
2003 \text { Spain, [32] }\end{array}$ & $\mathrm{P}$ & ERS & 6 & 14 & 59 & $14 / 0$ & $40 \pm 3$ & 14 & 64 & $14 / 0$ & $41 \pm 4$ \\
\hline $\begin{array}{c}\text { Koechlin, } \\
\text { 2004 France, [33] }\end{array}$ & $\mathrm{P}$ & GOLD & 7 & 7 & 60 & $7 / 0$ & $48 \pm 3.9$ & 10 & 58 & $10 / 0$ & $45.1 \pm 2.7$ \\
\hline $\begin{array}{c}\text { Sergi, } \\
2006 \text { Italy, [35] }\end{array}$ & $\mathrm{P}$ & ERS & 7 & 46 & 77.7 & $46 / 0$ & $40.8 \pm 5.6$ & 40 & 75.7 & $40 / 0$ & $38.2 \pm 5.4$ \\
\hline $\begin{array}{c}\text { Yang, } \\
\text { 2006 China, [34] }\end{array}$ & $\mathrm{P}$ & ATS & 7 & 34 & 64.8 & $34 / 0$ & $41.6 \pm 4.1$ & 47 & 67.6 & $47 / 0$ & $40.8 \pm 2.9$ \\
\hline $\begin{array}{c}\text { Gonlugur, } \\
2007 \text { Turkey, [36] }\end{array}$ & $\mathrm{R}$ & GOLD & 6 & 133 & 60.9 & $103 / 30$ & $38.2 \pm 6.9$ & 450 & 61.9 & $348 / 102$ & $37.7 \pm 8.5$ \\
\hline $\begin{array}{l}\text { Coin, } \\
2010 \text { Italy, [37] }\end{array}$ & $\mathrm{P}$ & ERS & 7 & 41 & 76 & $41 / 0$ & $43 \pm 4.7$ & 41 & 75.7 & $41 / 0$ & $38.3 \pm 5.4$ \\
\hline $\begin{array}{c}\text { Garcia-Rio, } \\
2010 \text { Spain, [18] }\end{array}$ & $\mathrm{P}$ & GOLD & 7 & 110 & 55 & $51 / 59$ & $46.3 \pm 8.3^{\S, *}$ & 324 & 64 & $241 / 83$ & $44.9 \pm 7.5^{\S, *}$ \\
\hline $\begin{array}{c}\text { Doucet, } \\
\text { 2010 Canada, [39] }\end{array}$ & $\mathrm{P}$ & ATS & 6 & 7 & 60 & $2 / 5$ & $43 \pm 1$ & 12 & 60 & $8 / 4$ & $43 \pm 3$ \\
\hline $\begin{array}{c}\text { Garcia-Rio, } \\
\text { 2011 Spain, [38] }\end{array}$ & $\mathrm{P}$ & GOLD & 7 & 85 & - & - & $45.9 \pm 2.7$ & 173 & - & - & $45.9 \pm 2.7$ \\
\hline $\begin{array}{c}\text { Elsammak, } \\
2012 \text { Saudi Arabian, [40] }\end{array}$ & $\mathrm{P}$ & GOLD & 7 & 34 & 63.0 & $20 / 14$ & $42.2 \pm 2.1$ & 70 & 62.6 & $47 / 23$ & $35.1 \pm 4.8$ \\
\hline
\end{tabular}


Table 1. Cont.

\begin{tabular}{|c|c|c|c|c|c|c|c|c|c|c|c|}
\hline \multirow[b]{2}{*}{$\begin{array}{l}\text { First Author, } \\
\text { Year Country }\end{array}$} & \multirow[b]{2}{*}{$\begin{array}{l}\text { Study } \\
\text { Design }\end{array}$} & \multirow[b]{2}{*}{ Diagnosis } & \multirow[b]{2}{*}{$\begin{array}{l}\text { NOS } \\
\text { (Stars) }\end{array}$} & \multicolumn{4}{|c|}{ Control Group } & \multicolumn{4}{|c|}{ COPD Group } \\
\hline & & & & $n$ & $\begin{array}{c}\text { Age } \\
\text { (Years) }\end{array}$ & $\begin{array}{l}\text { Gender } \\
(\mathrm{M} / \mathrm{F})\end{array}$ & $\begin{array}{c}\text { Albumin } \\
\text { Mean } \pm \text { SD }\end{array}$ & $n$ & $\begin{array}{c}\text { Age } \\
\text { (Years) }\end{array}$ & $\begin{array}{c}\text { Gender } \\
(\mathrm{M} / \mathrm{F})\end{array}$ & $\begin{array}{c}\text { Albumin } \\
\text { Mean } \pm \text { SD }\end{array}$ \\
\hline $\begin{array}{c}\text { Ye, } \\
2012 \text { China, [41] }\end{array}$ & $\mathrm{P}$ & GOLD & 8 & 20 & 67.5 & $15 / 5$ & $42.4 \pm 3.4$ & 43 & 68.4 & $40 / 3$ & $36.8 \pm 4.3$ \\
\hline $\begin{array}{c}\text { Jackson, } \\
2013 \text { England, [42] }\end{array}$ & $\mathrm{P}$ & GOLD & 9 & 100 & 63 & $59 / 41$ & $39.0 \pm 5.1$ & 104 & 65 & $60 / 44$ & $37.6 \pm 5.7$ \\
\hline $\begin{array}{c}\text { Elmahallawy, } \\
2013 \text { Egypt, [43] }\end{array}$ & $\mathrm{P}$ & GOLD & 7 & 300 & 64.7 & $138 / 162$ & $34.0 \pm 6.1$ & 300 & 65 & $152 / 148$ & $33.5 \pm 7$ \\
\hline $\begin{array}{l}\text { Emami Ardestani, } \\
2015 \text { Iran, [44] }\end{array}$ & $\mathrm{P}$ & GOLD & 6 & 120 & 43.7 & $120 / 0$ & $42 \pm 5.55$ & 60 & 59.1 & $60 / 0$ & $41 \pm 5.7$ \\
\hline $\begin{array}{l}\text { Milevoj Kopčinović, } \\
\text { 2016 Croatia, [45] }\end{array}$ & $\mathrm{P}$ & GOLD & 7 & 45 & 58.0 & $20 / 25$ & $49.2 \pm 6.9$ & 106 & 64.3 & $80 / 26$ & $39.3 \pm 5.8$ \\
\hline $\begin{array}{c}\text { Ozgul, } \\
2017 \text { Turkey, [46] }\end{array}$ & $\mathrm{P}$ & GOLD & 9 & 210 & 57.4 & $119 / 91$ & $35 \pm 12$ & 175 & 61 & $110 / 65$ & $33 \pm 5$ \\
\hline $\begin{array}{c}\text { Terashima, } \\
\text { 2017 Japan, [47] }\end{array}$ & $\mathrm{P}$ & GOLD & 6 & 76 & 65.1 & $49 / 27$ & $43.7 \pm 2.1$ & 60 & 73.9 & $55 / 5$ & $41.3 \pm 3.5$ \\
\hline $\begin{array}{c}\text { Lenártová, } \\
\text { 2017 Slovakia, [19] }\end{array}$ & $\mathrm{P}$ & GOLD & 7 & 60 & - & - & $41.7 \pm 2.1^{\S, *}$ & 60 & - & - & $39.7 \pm 3.5 \S$ \\
\hline $\begin{array}{c}\text { Omote, } \\
\text { 2017 Japan, [48] }\end{array}$ & $\mathrm{R}$ & GOLD & 7 & 42 & 63 & $32 / 10$ & $36 \pm 6$ & 43 & 67 & $37 / 6$ & $36 \pm 5$ \\
\hline $\begin{array}{c}\text { Cao, } \\
2017 \text { China, [49] }\end{array}$ & $\mathrm{P}$ & GOLD & 6 & 33 & 70 & $26 / 7$ & $45.3 \pm 2.3$ & 33 & 73 & $26 / 7$ & $37.8 \pm 5.8$ \\
\hline $\begin{array}{c}\text { Kuo, } \\
2019 \text { Taiwan, [50] }\end{array}$ & $\mathrm{P}$ & GOLD & 6 & 44 & 53.3 & $36 / 8$ & $44 \pm 3$ & 75 & 71.5 & $67 / 8$ & $43 \pm 4$ \\
\hline $\begin{array}{l}\text { Janciauskiene, } \\
2020 \text { Spain, [51] }\end{array}$ & $\mathrm{P}$ & GesEPOC & 7 & 312 & 55 & $138 / 174$ & $46 \pm 4.7$ & 303 & 64 & $223 / 80$ & $45 \pm 3.8$ \\
\hline
\end{tabular}

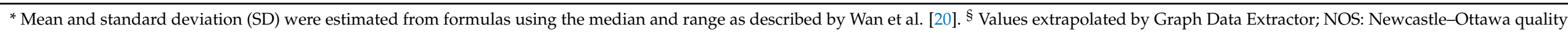
assessment scale for case-control studies. $\mathrm{P}=$ prospective; $\mathrm{R}=$ retrospective. 


\section{Discussion}

COPD is characterized by the presence of persistent respiratory symptoms and a progressive airflow limitation caused by exposure to noxious particles. The GOLD definition of COPD focuses on the association of the airflow limitation with the inflammatory response of the lung that, in patients with COPD, seems to be altered [1]. Inflammation in COPD is characterized by excess alveolar macrophages, neutrophils, and $\mathrm{T}$ lymphocytes that secrete a variety of proinflammatory mediators, including cytokines, chemokines, and growth factors [6]. Oxidative stress is thought to play a key role in driving COPD-related inflammation. In fact, both exogenous ROS and those released by activated inflammatory and structural cells might lead to the activation of proinflammatory molecules, to lipid, protein, and DNA damage, and to corticosteroid resistance through the inactivation of histone deacetylase 2 [6]. This prevents corticosteroids from inactivating inflammatory genes. Moreover, oxidative stress suppresses nuclear factor erythroid 2-related factor 2 (Nrf2) activity, resulting in reduced antioxidant gene expression [52]. In addition, COPD patients exhibit a systemic proinflammatory state characterized by an increase in circulating cytokines, chemokines, and acute-phase proteins [53]. Moreover, in these patients, the pro-oxidant mechanisms overcome the antioxidant defenses with consequent oxidative stress [12,54]. Albumin is a multifunctional plasma protein that is described to diminish in inflammatory conditions, and it is considered a negative acute-phase protein [11]. Moreover, albumin has important antioxidant properties being the major extracellular source of sulfhydryl groups, which represents an important first defense system against oxidative stress [55]. We have recently reported a reduction in plasma protein sulfhydryl groups in COPD patients compared to healthy controls [56]. In addition, reduced levels of antioxidants have been extensively reported in patients with COPD, as mentioned before [54]. Another important role of albumin is that of a clinical biomarker of malnutrition [12]. Hypoalbuminemia can be caused by poor food intake/absorption, older age, the presence of comorbidities, and proinflammatory cytokines that inhibit albumin production. The influence of these factors is accumulative on the risk of hypoalbuminemia [57]. In patients with COPD, these factors often co-exist. For example, the prevalence of COPD increases with age, peaking after sixty years of age [1]. Moreover, it frequently occurs in association with several comorbidities $[58,59]$ and with the progressive decline in exercise capacity, due to skeletal muscle wasting in older people. In turn, this is due to an imbalance between protein synthesis and breakdown. Malnutrition and weight loss are common among people with COPD and certain metabolic characteristics, such as a depleted fat mass and fat-free mass, can further impact on the loss of lung function capacity $[13,60]$. These features may be observed in stable COPD irrespectively of the degree of airflow limitation [61]. Our meta-analysis showed that overall, serum albumin concentrations were significantly lower in COPD patients compared to non-COPD controls. However, there was significant heterogeneity and publication bias, even if trim-and-fill analysis found that no study was missing or should be added. Age, sex, BMI, FEV1, FEV1/FVC, CRP, the continent where the study was conducted, and the year of publication did not contribute to this heterogeneity or to the pooled SMD. Conversely, the different guidelines used for COPD diagnosis contributed to the observed heterogeneity. We were not able to consider other factors, as they were not discussed in individual studies. These factors include the proportion of former and current smokers, the use of specific medications, and pre-analytical and analytical factors, such as sample storage, timing, conditions, and procedures for analysis. Moreover, three studies included in the meta-analysis comprised non-healthy subjects in the non-COPD group. Although albumin concentrations might have been theoretically affected, the SMD remained virtually unchanged after removing these three studies. Another limitation is represented by the relatively small number of subjects in a significant number of studies, which limits the representativity of the sample in terms of variability in disease phenotype and severity. This might have also explained the lack of significant differences after sorting for disease severity. Despite these limitations, our meta-analysis has the merit of comprehensively reviewing, for the first time, the available evidence on this topic, serving as a robust refer- 
ence for future studies. In conclusion, our review and meta-analysis showed that serum albumin concentrations were significantly lower in patients with stable COPD compared to non-COPD subjects. This supports the presence of a deficit in systemic anti-inflammatory and antioxidant defense mechanisms, as well as malnutrition, in COPD. However, the significant heterogeneity of the studies assessed warrants further investigations with rigorous methods and standardized diagnostic criteria.

Author Contributions: Study design, E.Z., A.Z., P.P.; data collection, E.S., S.M., A.A.M., A.Z.; data analysis, A.G.F., E.S., S.M., C.C., A.Z.; writing-original draft, E.Z., P.P.; writing-review and editing, E.Z., A.G.F., A.A.M., P.P.; supervision, E.Z., A.Z., P.P. All authors have read and agreed to the published version of the manuscript.

Funding: This research received no external funding.

Institutional Review Board Statement: Not applicable.

Informed Consent Statement: Not applicable.

Data Availability Statement: No new data were created or analyzed in this study. Data sharing is not applicable to this article.

Conflicts of Interest: The authors declare no conflict of interest.

\section{References}

1. Vogelmeier, C.F.; Criner, G.J.; Martinez, F.J.; Anzueto, A.; Barnes, P.J.; Bourbeau, J.; Celli, B.R.; Chen, R.; Decramer, M.; Fabbri, L.M.; et al. Global Strategy for the Diagnosis, Management, and Prevention of Chronic Obstructive Lung Disease 2017 Report. GOLD Executive Summary. Am. J. Respir Crit Care Med. 2017, 195, 557-582. [CrossRef]

2. Adeloye, D.; Chua, S.; Lee, C.; Basquill, C.; Papana, A.; Theodoratou, E.; Nair, H.; Gasevic, D.; Sridhar, D.; Campbell, H.; et al. Global and regional estimates of COPD prevalence: Systematic review and meta-analysis. J. Glob Health 2015, 5, 020415. [CrossRef]

3. Lozano, R.M.; Naghavi, M.; Foreman, K.; Lim, S.; Shibuya, K.; Aboyans, V.; Abraham, J.; Adair, T.; Aggarwal, R.; Ahn, S.Y.; et al. Global and regional mortality from 235 causes of death for 20 age groups in 1990 and 2010: A systematic analysis for the Global Burden of Disease Study 2010. Lancet 2012, 380, 2095-2128. [CrossRef]

4. Postma, D.S.; Bush, A.; van den Berge, M. Risk factors and early origins of chronic obstructive pulmonary disease. Lancet 2015, 385, 899-909. [CrossRef]

5. Salvi, S.S.; Barnes, P.J. Chronic obstructive pulmonary disease in non-smokers. Lancet 2009, 374, 733-743. [CrossRef]

6. Barnes, P.J. Inflammatory Mechanisms in Patients with Chronic Obstructive Pulmonary Disease. J. Allergy Clin. Immunol. 2016, 138, 16-27. [CrossRef] [PubMed]

7. Kirkham, P.A.; Barnes, P.J. Oxidative stress in COPD. Chest 2013, 144, 266-273. [CrossRef]

8. Quinlan, G.J.; Martin, G.S.; Evans, T.W. Albumin: Biochemical properties and therapeutic potential. Hepatology 2005, 41, 1211-1219. [CrossRef]

9. Oettl, K.; Stauber, R.E. Physiological and pathological changes in the redox state of human serum albumin critically influence its binding properties. Br. J. Pharmacol. 2007, 151, 580-590. [CrossRef]

10. Roche, M.; Rondeau, P.; Singh, N.R.; Tarnus, E.; Bourdon, E. The antioxidant properties of serum albumin. FEBS Lett. 2008, 582, 1783-1787. [CrossRef]

11. Gabay, C.; Kushner, I. Acute-phase proteins and other systemic responses to inflammation. N. Engl. J. Med. 1999, $340,448-454$. [CrossRef]

12. Zhang, Z.; Pereira, S.L.; Luo, M.; Matheson, E.M. Evaluation of Blood Biomarkers Associated with Risk of Malnutrition in Older Adults: A Systematic Review and Meta-Analysis. Nutrients 2017, 9, 829. [CrossRef] [PubMed]

13. Nguyen, H.T.; Collins, P.F.; Pavey, T.G.; Nguyen, N.V.; Pham, T.D.; Gallegos, D.L. Nutritional status, dietary intake, and health-related quality of life in outpatients with COPD. Int. J. Chron. Obstruct. Pulmon. Dis. 2019, 14, 215-226. [CrossRef]

14. Wang, Y.; Stavem, K.; Dahl, F.A.; Humerfelt, S.; Haugen, T. Factors associated with a prolonged length of stay after acute exacerbation of chronic obstructive pulmonary disease (AECOPD). Int. J. Chron. Obstruct. Pulmon. Dis. 2014, 9, 99-105. [CrossRef] [PubMed]

15. Chen, C.W.; Chen, Y.Y.; Lu, C.L.; Chen, S.C.; Chen, Y.J.; Lin, M.S.; Chen, W. Severe hypoalbuminemia is a strong independent risk factor for acute respiratory failure in COPD: A nationwide cohort study. Int. J. Chron. Obstruct. Pulmon. Dis. 2015, 10, 1147-1154. [CrossRef] [PubMed]

16. Hasegawa, W.; Yamauchi, Y.; Yasunaga, H.; Sunohara, M.; Jo, T.; Matsui, H.; Fushimi, K.; Takami, K.; Nagase, T. Factors affecting mortality following emergency admission for chronic obstructive pulmonary disease. BMC Pulm. Med. 2014, 14, 151. [CrossRef] [PubMed] 
17. Wells, G.; Shea, B.; O'Connell, D.; Peterson, J.; Welch, V.; Losos, M.; Tugwell, P. The Newcastle-Ottawa Scale (NOS) for Assessing the Quality of Nonrandomised Studies in Meta-Analyses. 2013. Available online: http://www.ohri.ca/programs/clinical_ epidemiology / oxford.asp (accessed on 12 January 2021).

18. Garcia-Rio, F.; Miravitlles, M.; Soriano, J.B.; Muñoz, L.; Duran-Tauleria, E.; Sánchez, G.; Sobradillo, V.; Ancochea, J.; EPI-SCAN Steering Committee. Systemic inflammation in chronic obstructive pulmonary disease: A population-based study. Respir. Res. 2010, 11, 63.

19. Lenártová, P.; Kopčeková, J.; Gažarová, M.; Mrázová, J.; Wyka, J. Biochemical parameters as monitoring markers of the inflammatory reaction by patients with chronic obstructive pulmonary disease (COPD). Rocz Panstw Zakl Hig. 2017, 68, 185-190.

20. Wan, X.; Wang, W.; Liu, J.; Tong, T. Estimating the sample mean and standard deviation from the sample size, median, range and/or interquartile range. BMC Med. Res. Methodol. 2014, 14, 135. [CrossRef]

21. Bowden, J.; Tierney, J.F.; Copas, A.J.; Burdett, S. Quantifying, displaying and accounting for heterogeneity in the meta-analysis of RCTs using standard and generalised Q statistics. BMC Med. Res. Methodol. 2011, 11, 41. [CrossRef]

22. Higgins, J.P.; Thompson, S.G. Quantifying heterogeneity in a meta-analysis. Stat. Med. 2002, 21, 1539-1558. [CrossRef] [PubMed]

23. Tobias, A. Assessing the influence of a single study in the meta-analysis estimate. Stata Tech. Bull. 1999, $47,15-17$.

24. Begg, C.B.; Mazumdar, M. Operating characteristics of a rank correlation test for publication bias. Biometrics 1994, 50, 1088-1101. [CrossRef] [PubMed]

25. Sterne, J.A.; Egger, M. Funnel plots for detecting bias in meta-analysis: Guidelines on choice of axis. J. Clin. Epidemiol. 2001, 54, 1046-1055. [CrossRef]

26. Duval, S.; Tweedie, R. Trim and fill: A simple funnel-plot-based method of testing and adjusting for publication bias in meta-analysis. Biometrics 2000, 56, 455-463. [CrossRef] [PubMed]

27. Liberati, A.; Altman, D.G.; Tetzlaff, J.; Mulrow, C.; Gøtzsche, P.C.; Ioannidis, J.P.; Clarke, M.; Devereaux, P.J.; Kleijnen, J.; Moher, D. The PRISMA statement for reporting systematic reviews and meta-analyses of studies that evaluate healthcare interventions: Explanation and elaboration. BMJ 2009, 339, b2700. [CrossRef]

28. Fiaccadori, E.; Coffrini, E.; Fracchia, C.; Rampulla, C.; Montagna, T.; Borghetti, A. Hypophosphatemia and phosphorus depletion in respiratory and peripheral muscles of patients with respiratory failure due to COPD. Chest 1994, 105, 1392-1398. [CrossRef]

29. Paiva, S.A.; Godoy, I.; Vannucchi, H.; Fávaro, R.M.; Geraldo, R.R.; Campana, A.O. Assessment of vitamin A status in chronic obstructive pulmonary disease patients and healthy smokers. Am. J. Clin. Nutr. 1996, 64, 928-934. [CrossRef]

30. Dahlén, I.; Lindberg, E.; Janson, C.; Stâlenheim, G. Delayed type of hypersensitivity and late allergic reactions in patients with stable COPD. Chest 1999, 116, 1625-1631. [CrossRef]

31. Yoneda, T.; Yoshikawa, M.; Fu, A.; Tsukaguchi, K.; Okamoto, Y.; Takenaka, H. Plasma levels of amino acids and hypermetabolism in patients with chronic obstructive pulmonary disease. Nutrition 2001, 17, 95-99. [CrossRef]

32. Hernández, N.; Orozco-Levi, M.; Belalcázar, V.; Pastó, M.; Minguella, J.; Broquetas, J.M.; Gea, J. Dual morphometrical changes of the deltoid muscle in patients with COPD. Respir. Physiol. Neurobiol. 2003, 134, 219-229. [CrossRef]

33. Koechlin, C.; Couillard, A.; Cristol, J.P.; Chanez, P.; Hayot, M.; Le Gallais, D.; Préfaut, C. Does systemic inflammation trigger local exercise-induced oxidative stress in COPD? Eur. Respir. J. 2004, 23, 538-544. [CrossRef]

34. Yang, Y.M.; Sun, T.Y.; Liu, X.M. The role of serum leptin and tumor necrosis factor-alpha in malnutrition of male chronic obstructive pulmonary disease patients. Chin. Med. J. (Engl.) 2006, 119, 628-633. [CrossRef] [PubMed]

35. Sergi, G.; Coin, A.; Marin, S.; Vianello, A.; Manzan, A.; Peruzza, S.; Inelmen, E.M.; Busetto, L.; Mulone, S.; Enzi, G. Body composition and resting energy expenditure in elderly male patients with chronic obstructive pulmonary disease. Respir. Med. 2006, 100, 1918-1924. [CrossRef] [PubMed]

36. Gonlugur, U.; Gonlugur, T.A. Retrospective Analysis of Nutritional Parameters in Chronic Obstructive Pulmonary Disease between Sexes. J. Clin. Biochem. Nutr. 2007, 41, 175-178. [CrossRef] [PubMed]

37. Coin, A.; Sergi, G.; Marin, S.; Vianello, A.; Perissinotto, E.; Sarti, S.; Rinaldi, G.; Mosele, M.; Inelmen, E.M.; Enzi, G.; et al. Predictors of low bone mineral density in elderly males with chronic obstructive pulmonary disease: The role of body mass index. Aging Male 2010, 13, 142-147. [CrossRef]

38. García-Rio, F.; Soriano, J.B.; Miravitlles, M.; Muñoz, L.; Duran-Tauleria, E.; Sánchez, G.; Sobradillo, V.; Ancochea, J. Overdiagnosing subjects with COPD using the 0.7 fixed ratio: Correlation with a poor health-related quality of life. Chest 2011, 139, 1072-1080. [CrossRef]

39. Doucet, M.; Dubé, A.; Joanisse, D.R.; Debigaré, R.; Michaud, A.; Paré, M.Ė.; Vaillancourt, R.; Fréchette, E.; Maltais, F. Atrophy and hypertrophy signalling of the quadriceps and diaphragm in COPD. Thorax 2010, 65, 963-970. [CrossRef]

40. Elsammak, M.Y.; Attia, A.; Suleman, M. Fibroblast growth factor 23 (FGF23) and hypophosphatemia in patients with COPD. J. Med. Biochem. 2012, 31, 12-18. [CrossRef]

41. Ye, M.; Yu, H.; Yu, W.; Zhang, G.; Xiao, L.; Zheng, X.; Wu, J. Evaluation of the significance of circulating insulin-like growth factor-1 and C-reactive protein in patients with chronic obstructive pulmonary disease. J. Int. Med. Res. 2012, 40, 1025-1035. [CrossRef]

42. Jackson, A.S.; Shrikrishna, D.; Kelly, J.L.; Kemp, S.V.; Hart, N.; Moxham, J.; Polkey, M.I.; Kemp, P.; Hopkinson, N.S. Vitamin D and skeletal muscle strength and endurance in COPD. Eur. Respir J. 2013, 41, 309-316. [CrossRef] [PubMed]

43. Elmahallawy, I.I.; Qora, M.A. Prevalence of chronic renal failure in COPD patients. Egypt J. Chest Dis. Tuberc. 2013, 62, 221-227. [CrossRef] 
44. Emami Ardestani, M.; Zaerin, O. Role of Serum Interleukin 6, Albumin and C-Reactive Protein in COPD Patients. Tanaffos 2015, 14, 134-140. [PubMed]

45. Milevoj Kopčinović, L.; Domijan, A.M.; Posavac, K.; Čepelak, I.; Žanić Grubišić, T.; Rumora, L. Systemic redox imbalance in stable chronic obstructive pulmonary disease. Biomarkers 2016, 21, 692-698. [CrossRef] [PubMed]

46. Ozgul, G.; Seyhan, E.C.; Özgül, M.A.; Günlüoğlu, M.Z. Red Blood Cell Distribution Width in Patients With Chronic Obstructive Pulmonary Disease and Healthy Subjects. Arch Bronconeumol. 2017, 53, 107-113. [CrossRef]

47. Terashima, T.; Chubachi, S.; Matsuzaki, T.; Nakajima, T.; Satoh, M.; Iwami, E.; Yoshida, K.; Katakura, A.; Betsuyaku, T. The association between dental health and nutritional status in chronic obstructive pulmonary disease. Chron. Respir. Dis. 2017, 14, 334-341. [CrossRef]

48. Omote, N.; Hashimoto, N.; Morise, M.; Sakamoto, K.; Miyazaki, S.; Ando, A.; Nakahara, Y.; Hasegawa, Y. Impact of mild to moderate COPD on feasibility and prognosis in non-small cell lung cancer patients who received chemotherapy. Int. J. Chron. Obstruct. Pulmon. Dis. 2017, 12, 3541-3547. [CrossRef]

49. Cao, T.; Xu, N.; Wang, Z.; Liu, H. Effects of Glutathione S-Transferase Gene Polymorphisms and Antioxidant Capacity per Unit Albumin on the Pathogenesis of Chronic Obstructive Pulmonary Disease. Oxid Med. Cell Longev. 2017, 2017, 6232397. [CrossRef]

50. Kuo, W.K.; Liu, Y.C.; Chu, C.M.; Hua, C.C.; Huang, C.Y.; Liu, M.H.; Wang, C.H. Amino Acid-Based Metabolic Indexes Identify Patients With Chronic Obstructive Pulmonary Disease And Further Discriminates Patients In Advanced BODE Stages. Int. J. Chron. Obstruct. Pulmon. Dis. 2019, 14, 2257-2266. [CrossRef]

51. Janciauskiene, S.; DeLuca, D.S.; Barrecheguren, M.; Welte, T.; Miravitlles, M.; Scientific Committee; Participating sites and coordinators. Serum Levels of Alpha1-antitrypsin and Their Relationship with COPD in the General Spanish Population. Arch. Bronconeumol. 2020, 56, 76-83. [CrossRef]

52. Mercado, N.; Thimmulappa, R.; Thomas, C.M.R.; Fenwick, P.S.; Chana, K.K.; Donnelly, L.E.; Biswal, S.; Ito, K.; Barnes, P.J. Decreased histone deacetylase 2 impairs Nrf2 activation by oxidative stress. Biochem. Biophys. Res. Commun. 2011, 406, 292-298. [CrossRef] [PubMed]

53. Su, B.; Liu, T.; Fan, H.; Chen, F.; Ding, H.; Wu, Z.; Wang, H.; Hou, S. Inflammatory Markers and the Risk of Chronic Obstructive Pulmonary Disease: A Systematic Review and Meta-Analysis. PLoS ONE 2016, 11, e0150586. [CrossRef] [PubMed]

54. Zinellu, E.; Zinellu, A.; Fois, A.G.; Fois, S.S.; Piras, B.; Carru, C.; Pirina, P. Reliability and Usefulness of Different Biomarkers of Oxidative Stress in Chronic Obstructive Pulmonary Disease. Oxid. Med. Cell Longev. 2020, 2020, 4982324. [CrossRef] [PubMed]

55. Rossi, R.; Giustarini, D.; Milzani, A.; Dalle-Donne, I. Cysteinylation andhomocysteinylation of plasma protein thiols during ageing ofhealthy human beings. J. Cell Mol. Med. 2009, 13, 3131-3140. [CrossRef]

56. Zinellu, A.; Fois, A.G.; Sotgia, S.; Zinellu, E.; Bifulco, F.; Pintus, G.; Mangoni, A.A.; Carru, C.; Pirina, P. Plasma protein thiols: An early marker of oxidative stress in asthma and chronic obstructive pulmonary disease. Eur. J. Clin. Invest. 2016, 46, 181-188. [CrossRef]

57. Cabrerizo, S.; Cuadras, D.; Gomez-Busto, F.; Artaza-Artabe, I.; Marín-Ciancas, F.; Malafarina, V. Serum albumin and health in older people: Review and meta-analysis. Maturitas 2015, 81, 17-27. [CrossRef]

58. Baty, F.; Putora, P.M.; Isenring, B.; Blum, T.; Brutsche, M. Comorbidities and Burden of COPD: A Population Based Case-Control Study. PLoS ONE 2013, 8, e63285. [CrossRef]

59. Divo, M.J.; Cote, C.; de Torres, J.P.; Casanova, C.; Marin, J.M.; Pinto-Plata, V.; Zulueta, J.; Cabrera, C.; Zagaceta, J.; Hunninghake, G.; et al. Comorbidities and risk of mortality in patients with chronic obstructive pulmonary disease. Am. J. Respir Crit. Care Med. 2012, 186, 155-161. [CrossRef]

60. Collins, P.F.; Yang, I.A.; Chang, Y.C.; Vaughan, A. Nutritional support in chronic obstructive pulmonary disease (COPD): An evidence update. J. Thorac. Dis. 2019, 11, S2230-S2237. [CrossRef]

61. Pirabbasi, E.; Najafiyan, M.; Cheraghi, M.; Shahar, S.; Abdul Manaf, Z.; Rajab, N.; Abdul Manap, R. Predictors' factors of nutritional status of male chronic obstructive pulmonary disease patients. ISRN Nurs. 2012, 2012, 782626. [CrossRef] 